\title{
Changes in percutaneous patent foramen ovale closure after the 2019 consensus: single center analysis of referrals and indications
}

\author{
(DIrzal Hadžibegović1*, \\ (D)Mario Sičaja², \\ (DIvana Jurin', \\ DJasmina Ćatić', \\ (D)Sandra Jakšić \\ Jurinjak ${ }^{3}$, \\ Neven Čače ${ }^{4}$
}

'University Hospital Dubrava, Zagreb, Croatia

${ }^{2}$ IImtal Klinik GmnH, Pfaffenhofen an der Ilm, Deutschland

${ }^{3}$ University Hospital Centre Zagreb, Zagreb, Croatia

${ }^{4}$ University Hospital Centre Rijeka, Rijeka, Croatia
RECEIVED:

December 6, 2020

ACCEPTED:

December 18, 2020

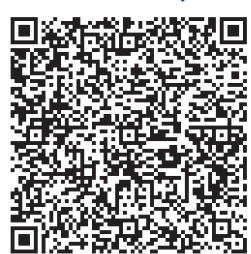

KEYWORDS: cryptogenic stroke, patent foramen ovale, closure device.

CITATION: Cardiol Croat. 2021;16(1-2):41 | https://doi.org/10.15836/ccar2021.41

*ADDRESS FOR CORRESPONDENCE: Irzal Hadžibegović, Klinička bolnica Dubrava, Avenija Gojka Šuška 6, HR-10000 Zagreb, Croatia. / Phone: +385-91-5333091 / E-mail: irzalh@gmail.com

ORCID: Irzal Hadžibegović, https://orcid.org/0000-0002-3768-9134 • Mario Sičaja, https://orcid.org/0000-0003-0773-4720 Ivana Jurin, https://orcid.org/0000-0002-2637-9691 • Jasmina Ćatić, https://orcid.org/0000-0001-6582-4201

Sandra Jakšić Jurinjak, https://orcid.org/0000-0002-7349-6137

IIIIIIIIIIIIIIIIIIIIIIIIIIIIIIIIIIIIIIIIIIIIIIIIIIIIIIIIIIIIIIIIIIIIIIIIIIIIIIIIIIIIIIIIIIIIIIIIIIIIIIIIIIIIIIIIIIII

Background: In April 2019 SCAI published the first consensus document on patent foramen ovale (PFO) closure after cryptogenic stroke, driven by the positive results of CLOSE, REDUCE, and DEFENSE-PFO trials. ${ }^{1}$ Aim: To analyze referral and indication for PFO closure in our institution before and after April 2019.

Methods: We analyzed clinical characteristics, imaging data, referral information and follow-up data of patients that underwent PFO closure before and after the publication of 2019 consensus document.

Results: From 2016 to 2020 a total of 26 patients underwent PFO closure (14 patients before, and 12 patients after the 2019 consensus paper). There were no significant differences in patient's demographic and clinical characteristics. Patients in both groups were all referred by a neurologist after stroke/ TIA and underwent CT or MRI brain scan, 24-h ECG monitoring and TEE exam before the procedure. All patients treated after the 2019 consensus had subcortical lesions on CT or MRI. Only 1 patient in pre-consensus group with history of TIA had no imaging evidence of subcortical ischemia before the procedure. All patients had and at least 1 TEE high risk feature. There were more patients on direct oral anticoagulation drugs after stroke after the 2019 consensus. All patients were treated with Amplatzer device and received dual antiplatelet therapy for at least 3 months, with additional ASA continued indefinitely in selected high-risk patients. There were no documented recurrent ischemic events or new onset atrial fibrillation within 1 year after PFO closure in both groups.

Conclusion: There were no significant differences in referral and indications for PFO closure before and after the 2019 consensus. Very careful patient selection, in concordance with the consensus, relates to procedure safety and long-term outcomes. Neurologists remain the gatekeepers, whereas cardiologists plan and perform the procedure, and tailor postprocedural antithrombotic therapy. We observed an increase in annual volume after the consensus, most probably because of increased awareness after 2018, and facilitation of the decision to perform PFO closure after cryptogenic stroke brought by the consensus document.

LITERATURE IIIIIIIIIIIIIIIIIIIIIIIIIIIIIIIIIIIIIIIIIIIIIIIIIIIIIIIIIIIIIIIIIIIIIIIIIIIIIIIIIIIIIIIIIIIIIIIIIII

1. Safouris A, Kargiotis 0 , Psychogios K, Kalyvas P, Ikonomidis I, Drakopoulou M, Toutouzas K, Tsivgoulis G. A Narrative and Critical Review of Randomized-Controlled Clinical Trials on Patent Foramen Ovale Closure for Reducing the Risk of Stroke Recurrence. Front Neurol. 2020 Jun 4;11:434. https://doi.org/10.3389/fneur.2020.00434 